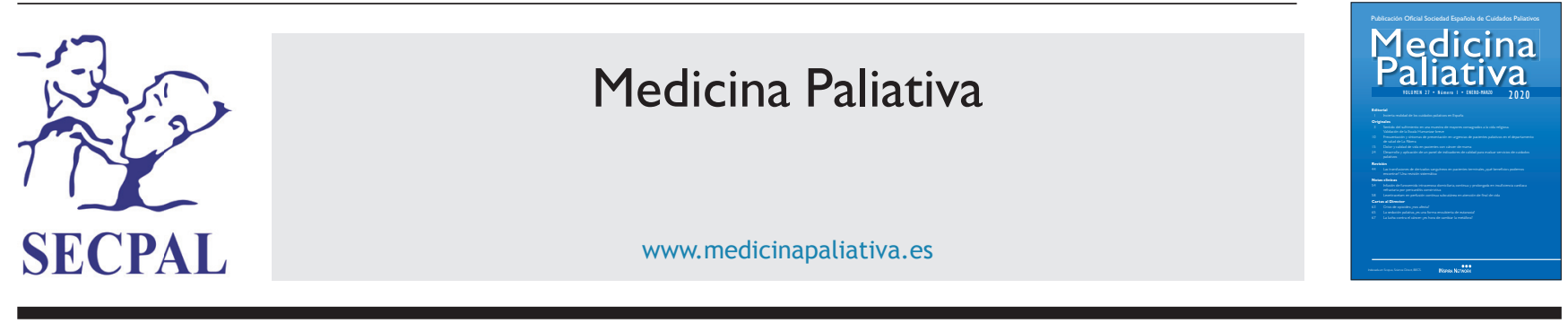

\title{
Incierta realidad de los cuidados paliativos en España
}

Las dificultades que presentan los profesionales sanitarios para atender a los pacientes paliativos son actualmente una realidad. El envejecimiento de la población y el creciente número de personas con enfermedades cronicodegenerativas y con cáncer representan un reto importante para los servicios de salud en las sociedades desarrolladas, donde el soporte familiar es decreciente. Muchos de estos enfermos, al final de su vida, padecen un sufrimiento intenso y precisan una atención sanitaria y social que implica a todos los ámbitos asistenciales, sin embargo, son atendidos en distintos enclaves sociosanitarios y por diferentes profesionales cada vez más especializados en su campo, distinto del paliativo.

En la sociedad actual existe una demanda generalizada de atención centrada en la persona, de calidad y a costes razonables, que permita una vida y una muerte dignas. Los cuidados paliativos (CP) son una parte esencial del manejo clínico de pacientes con cáncer ${ }^{1}$ y con determinadas enfermedades cronicodegenerativas ${ }^{2}$. Dichos CP tienen, entre otros, los objetivos de aliviar el sufrimiento así como de mejorar la calidad de vida y el proceso de morir de las personas, en una sociedad cada vez más individualista y con familias más pequeñas y aisladas. Este hecho se pone de manifiesto ya que el tamaño medio del hogar en España en 2017 fue de 2,49 personas y el 25,4 \% del total de hogares está conformado por personas que viven solas ${ }^{3}$.

Estos pacientes, en multitud de ocasiones, son atendidos durante las etapas finales de su enfermedad en hospitales y por profesionales especializados en patologías agudas, que generalmente no disponen de un equipo de soporte para CP, que podría atender y minimizar las dificultades asistenciales a las que estos profesionales se enfrentan ${ }^{4}$.

Aproximadamente, el 50-60 \% de las personas fallecidas han recorrido un proceso de deterioro en el último año de su vida ${ }^{5}$. En España, durante 2015, el cáncer fue la tercera causa de ingreso hospitalario con un 11,4\%, aunque el primer motivo de mortalidad en este ámbito alcanzó el 24,2 \% (Instituto Nacional de Estadística). Asimismo, solo en las unidades de medicina interna se atienden el $14,5 \%$ de todos los ingresos hospitalarios, asumiendo un $9,5 \%$ de la mortalidad global.

Afortunadamente, en los últimos años se ha experimentado un avance importante en el manejo de pacientes paliativos, no obstante, siguen existiendo áreas de mejora entre las que se puede destacar: el fomento de la formación en medicina paliativa, la necesidad de un enfoque integral de los cuidados que cubra, además del control de síntomas, los aspectos emocionales, sociales y espirituales de los pacientes y de sus cuidadores o familiares, la necesidad de mejorar la accesibilidad de los CP para todas las personas que los necesiten, la promoción del trabajo multidisciplinar y la coordinación entre los distintos niveles asistenciales, servicios y profesionales implicados.

Ya en 1999, Benítez del Rosario y cols. ${ }^{6}$ encuentran que las principales dificultades percibidas por profesionales de atención primaria (AP), para el manejo de CP, incluían problemas de coordinación con la atención especializada, problemas en la organización interna de los equipos, dificultades en el suministro de opiáceos a los pacientes, dificultades para mantener la asistencia continuada las 24 horas del día y déficit de confianza por parte de los pacientes y familiares en la capacitación del profesional de AP. Según estudios recientes, muchos de ellos continúan enquistados en países occidentales, como es la falta de confianza en los profesionales dedicados a la prestación de $\mathrm{CP}^{7}$; mientras que en los países orientales se añaden otros como los escasos conocimientos y habilidades de comunicación en la atención a enfermos terminales de cáncer y sus familias, o la falta de especialistas en $\mathrm{CP}^{8}$. Otros autores, asimismo, han descrito recientemente que entre las dificultades más frecuentes percibidas por los profesionales se incluirían la falta de un adecuado soporte a familiares ${ }^{9}$, las barreras en la comunicación con pacientes y familiares ${ }^{1,4}$, la dificultad en el control de síntomas y la ausencia de una adecuada educación en medicina paliativa por los profesionales ${ }^{1,4,9,10}$. Hirooka y cols. ${ }^{9}$ incluyen, además, la ausencia de un adecuado soporte psicológico a los pacientes, y otros autores, como Moss y cols., una inadecuada financiación para los CP10.

En líneas generales, multitud de estudios previos se han centrado en la valoración de las actitudes de los profesionales respecto a los cuidados del paciente en situación terminal. Sin embargo, en reducidas ocasiones se han investigado otras áreas de los CP, a la vez que existe poca información sobre las propiedades métricas de los instrumentos de medida utilizados.

En relación con esto último, los autores han empleado una amplia diversidad de cuestionarios, en la mayoría de las ocasiones no validados, que valoran las dificultades percibidas por distintos profesionales sanitarios y ámbitos asistenciales 
como pueden ser: enfermeras con labor asistencial en hospitales, en domicilios y en unidades de cuidados intensivos ${ }^{1,9,10}$, y médicos con desarrollo profesional en hospitales, centros de APy unidades de cuidados intensivos ${ }^{9,10}$. No obstante, es escasa la literatura sobre cuestionarios validados en español que valoren las dificultades en la provisión de CP por parte de los profesionales sanitarios.

El análisis pormenorizado de dichas dificultades, a través de instrumentos fiables, permitiría establecer planes de mejora con estrategias concretas y adaptadas a cada realidad. Las evidencias científicas existentes muestran que es esencial la educación de los profesionales de la salud implicados en la atención a estos pacientes ${ }^{9}$. Esto podría conseguirse a través de un enfoque interdisciplinario². Para ello, se han desarrollado programas específicos en diferentes países, como el "Education for Physicians in End-of-Life Care (EPEC)" en Estados Unidos"11, el "Palliative Care Emphasis program on symptom management and Assessment for Continuous medical Education (PEACE)" y el "Outreach Palliative Care Trial of Integrated Regional Model (OPTIM) study", en Japón ${ }^{12}$.

Por todo lo expuesto anteriormente, se podría afirmar la existencia de una incierta realidad asistencial de los profesionales que proporcionan CP en España. Un diagnóstico preciso de sus dificultades requiere un cuestionario validado al respecto y administrado a los distintos profesionales que atienden a estos pacientes en los distintos ámbitos. De este modo, se optimizará la elección de estrategias que han demostrado su efectividad en resolver dichas dificultades y, por tanto, los CP que brindan dignidad a los últimos días de vida.

Sofía Vidal Serrano ${ }^{1}$, Sergio López Alonso ${ }^{2,3}$ y María del Rocío Fernández Ojeda1,3

${ }^{1}$ Servicio de Medicina, Hospital San Juan de Dios del Aljarafe, Sevilla, España. ${ }^{2}$ Dispositivo de Cuidados Críticos y Urgencias, Distrito Sanitario Málaga, Servicio Andaluz de Salud, Málaga, España. ${ }^{3}$ Centro Universitario de Enfermería "San Juan de Dios", Universidad de Sevilla, Bormujos, Sevilla, España.

\section{Bibliografía}

1. Nakazawa Y, Miyashita M, Morita T, Umeda M, Oyagi Y, Ogasawara T. The palliative care self-reported practices scale and the palliative care difficulties scale: reliability and validity of two scales evaluating self-reported practices and difficulties experienced in palliative care by health professionals. J Palliat Med. 2010;13:427-37.

2. Leclerc B-S, Lessard S, Bechennec C, le Gal E, Benoit S, Bellerose L. Attitudes toward death, dying, end-of-life palliative care, and interdisciplinary practice in long term care workers. J Am Med Dir Assoc. 2014;15:207-13.

3. Instituto Nacional de Estadística (INE). Encuesta continua de hogares 2016. Madrid. Ministerio de Economía, Industria y Competitividad. España [Consultado 7 Ene 2019]. Disponible en: htt://www.ine.es/prensa/ech_2017.ppdf

4. Kutner JS, Metcalfe T, Vu KO, Fink R, Nelson-Marten P, Armstrong JD, et al. Implementation of an ad hoc hospital-based palliative care consult service. J Pain Symptom Manage. 2004;28:526-8.

5. McNamara B, Rosenwax LK, Holman CDJ. A method for defining and estimating the palliative care population. J Pain Symptom Manage. 2006;32:5-12.

6. Benítez del Rosario MA, Salinas Martín A, Asensio Fraile A, Armas J. [Palliative care in primary care: the opinion of professionals]. Aten primaria. 1999;23:187-91.

7. Figueroa MI, Sepanski R, Goldberg SP, Shah S. Improving teamwork, confidence, and collaboration among members of a pediatric cardiovascular intensive care unit multidisciplinary team using simulation-based team training. Pediatr Cardiol. 2013;34:612-9.

8. Miyashita M, Nishida S, Koyama Y, Kimura R, Sasahara T, Shirai Y, et al. The current status of palliative care teams in Japanese university hospitals: a nationwide questionnaire survey. Support Care Cancer. 2007;15:801-6.

9. Hirooka K, Miyashita M, Morita T, Ichikawa T, Yoshida S, Akizuki N, et al. Regional medical professionals' confidence in providing palliative care, associated difficulties and availability of specialized palliative care services in Japan. Jpn J Clin Oncol. 2014;44:249-56.

10. Moss AH, Demanelis AR, Murray J, Jack J. Barriers to quality end-of-life care in West Virginia ICU units: physicians' and nurses' prospectives. W V Med J. 2005;101:200-4.

11. Robinson K, Sutton S, von Gunten CF, Ferris FD, Molodyko N, Martinez J, et al. Assessment of the Education for Physicians on End-of-Life Care (EPEC) Project. J Palliat Med. 2004;7:637-45.

12. Tsuneto S. Past, present, and future of palliative care in Japan. Jpn J Clin Oncol. 2013;43:17-21. 